\title{
Ethics of recommending natural health products as physicians
}

\author{
Adriana Cappelletti, Matthew Greenacre \\ Faculty Reviewer: Ana Ning, PhD (King's University College at Western University)
}

\section{INTRODUCTION}

In Canada, pharmaceutical drugs must undergo a rigorous, multiphase clinical trial process to obtain approval by Health Canada. ${ }^{1}$ Physicians prescribe these drugs based on their evidence of effectiveness and safety according to a Western scientific framework. This practice of evidence-based medicine (EBM) is coupled with clinical expertise and consideration of patients' values. ${ }^{2}$ Although pharmaceutical drugs are considered conventional medicine in Canada, over $70 \%$ of Canadians also use or replace these treatments with natural health products (NHPs), such as herbal products, vitamins, traditional medicine and homeopathic medicine. ${ }^{3,4}$ NHPs fall under the umbrella of complementary and alternative medicine (CAM), which encompasses natural and traditional therapies such as yoga and acupuncture that have been scientifically verified to varying degrees. NHPs appeal to Canadians based on their non- or minimally invasive nature, to which patients attribute a low-risk profile. Opposition to Big Pharma and scientific reductionism also contribute to NHPs' popularity. ${ }^{5}$ However, safety and efficacy are defined more broadly for NHP licensing approval than is the case for pharmaceutical drugs, with textbooks, theories, experiences and beliefs constituting sources of evidence. ${ }^{6}$ Proponents of CAM argue that this flexibility is necessary because trial methods of EBM, notably randomized controlled trials (RCTs), are incompatible with evaluating CAM. ${ }^{7,8}$ Conversely, sceptics of CAM question the effectiveness of these looser regulations, believing CAM research to be poorly funded, lacking in proper control groups and published in self-referring alternative medicine-specific journals. ${ }^{5,79}$ In the context of these opposing views, this article will compare definitions of evidence in CAM and biomedicine before exploring legal and moral dimensions around the question of whether it is ethical for physicians to recommend NHPs without conventional scientific evidence of clinical safety and effectiveness.

\section{EVIDENCE IN BIOMEDICINE AND CAM}

Evidence includes any information a person uses to determine how likely it is that a given proposition, such as an idea, is true. Epistemology, or theory of knowledge, is defined as the criteria one uses to decide what is acceptable and sufficient evidence to believe a proposition. ${ }^{10}$ Although there are many CAMs encompassing a wide variety of belief systems, they are distinguished from biomedicine by their epistemology. ${ }^{11,12}$ Fundamentally, biomedical practitioners make diagnoses and provide treatments based on both clinical experience and knowledge that has been verified by the scientific method. ${ }^{13}$ Similarly, CAM practitioners practice from their clinical experience and CAM theory. In contrast, theories that underlie CAM are accepted out of tradition, culture, identity, personal narrative or anecdotal evidence of healing, rather than experimental laboratory or population-level science. ${ }^{12,14,15}$ Using the results of an RCT, a biomedical practitioner is able to make predictions only about the drug effects and disease symptoms of the theoretical average patient who matches the criteria of the trial. Predictions can only be made for the length of time a participant was studied, which may be relatively short. Understanding of health and illness in CAM is based on the real world experience of the individual patient, considering their entire context and all symptoms from physical to their relationships with family and friends, with information usually collected over a long relationship with the practitioner. ${ }^{14,16}$

When both CAM and biomedicine describe a model of disease in an individual patient and recommend the consequent treatment, the explanation one believes depends on one's particular epistemology. However, mechanisms of action proposed by CAM theory are often epistemologically independent of pathophysiology..$^{12,17}$ For instance, some CAMs claim to work by restoring balance to the body's vital energy. Because such an energy field has not been shown to exist using the scientific method, biomedicine finds that explanation highly unlikely. ${ }^{11}$ A subjective experience of one's vital energy being restored by a treatment may provide ample evidence to convince one that such a force exists, but it would be a contradiction to accept the scientific account as well.

Though the explanation of how a CAM treatment is effective might differ greatly between a CAM theory and the biomedical model, biomedicine may consider it effective as long as it passes the scientific method. For instance, there is promising scientific evidence of acupuncture's effectiveness on smoking cessation, demonstrated by a meta-analysis of RCTs. ${ }^{18}$

The scientific method strives to provide the most accurate description of reality because it continually compares its descriptions to observations of the external world, rather than a particular cultural narrative or traditional belief. A proper explanation of the myriad reasons why science rarely fully achieves this objective ideal in practice is beyond the scope of this article, but can be summarized by saying that it is conducted by humans with history, culture, motivations and biases, rather than perfect hypothesis-generating and testing machines. ${ }^{12,19}$

Biomedicine remains the description of disease mechanisms and their treatment that is most closely aligned with objective, empirical measurement of reality. However, biomedicine is illequipped to determine what being sick means for a patient, or how a treatment may change their whole life. Subjective, holistic experience is used as evidence for determining meaning and purpose, something that most CAMs are adept at facilitating. ${ }^{12,14}$ 


\section{MORAL CONSIDERATIONS}

With regard to Beauchamp and Childress's 4 principles of medical ethics, arguments can be made both for and against recommending NHPs. ${ }^{20}$ First, consider autonomy. Discussing NHPs as a therapeutic option allows patients to make an informed decision about their care that includes the full range of options available to them. This empowerment psychologically benefits patients. ${ }^{5,19}$ The caveat is that patients may not actually be making informed decisions about their care if all existing evidence on NHPs is not disclosed to them, either due to insufficient research on their effects or if the practitioner is reluctant to reveal that treatments such as homeopathy do not show greater than a placebo effect in scientific trials. $^{21}$

Regarding the principle of nonmaleficence, sceptics of CAM fear that government regulation and physician recommendation of NHPs may provide "undue legitimacy" to therapies not grounded in science. ${ }^{5,22}$ This becomes especially worrisome where patients choose to replace rather than complement conventional medicine with alternative treatment. ${ }^{19,22}$ For example, in March 2013, Calgarian boy Ryan Lovett died of an easily treatable bacterial throat infection due to his mother's administration of homeopathic remedies instead of antibiotics. ${ }^{23}$ Despite this risk, government regulation of homeopathy and other forms of CAM can be viewed as a public safety measure, ensuring a standard of care and creating a formal body for complaints. ${ }^{22}$ Furthering this notion of beneficence is the idea of using CAM after attempting conventional therapies, as an "extra safeguard rather than a potential risk."

Considering justice, recommending use of NHPs without scientifically proven effectiveness may be unjust, as these therapies may incur a significant financial burden on patients for no more than a placebo effect. ${ }^{5}$

The utilitarian standpoint also does not present a definite argument, as utility is variably defined. For some, the utility of having control over one's care plan may outweigh the utility of obtaining the outcome of using conventional medicine, even if this means dying versus living. ${ }^{25}$ This notion of utility serves the individual; however, Canadian healthcare is publicly funded and thus utility to society must be considered. If a patient, initially using ineffective nonevidence-based therapies, turns to conventional medicine in late-stage disease, society bears the financial cost of this decision. ${ }^{5}$ However, this financial cost may be outweighed by the utility of living in a multicultural society that respects other groups' beliefs, even if this means that the economy is not maximized.

Lastly, in deontology, which evaluates morality based on duty, recommending CAM without scientific evidence may be morally right if the practitioner's intent is to help the patient, even if they inadvertently fail to account for potential risks. Nevertheless, physicians have an obligation mandated by the College of Physicians and Surgeons of Ontario (CPSO) to ensure that the care they are providing is grounded in EBM., ${ }^{56}$ The CPSO requires that all assessments, diagnoses and treatments must be informed by science, and physicians must never recommend therapies that have been proven to be ineffective through scientific study. ${ }^{26}$ As such, recom- mending therapy without good evidence of clinical effectiveness and safety may run counter to both the patient's best interests and a physicians' professional obligations.

\section{LEGAL ARGUMENTS}

In accordance with the policy of the CPSO, the Ontario government has committed to scientific, evidence-based medicine with the Excellent Care for All Act (2010), which establishes quality committees and the Ontario Health Quality Council that have a mandate to ensure that healthcare is supported by the best available scientific evidence. ${ }^{27}$

The elevated legal status of biomedicine in Ontario is dramatically illustrated by the fact that the Child and Family Services Act (1990) may make a child a ward of the Children's Aid Society if parents refuse to consent to a medical procedure that the court deems to be in the child's best interests. However, this legislation does give special protection to the rights of Ontario's indigenous peoples to determine what the best interests of a child are from their own cultural perspective. ${ }^{28}$ Hamilton Health Science Corp v. D.H. (2015) is a recent case of an application by a biomedical hospital for a court order to give chemotherapy to an 11-year-old girl with leukemia from the Haudenosaunee nation when she and her family chose to use traditional indigenous medicine instead. The application was denied by the Ontario Court of Justice. The court chose to uphold the cultural practices and worldview of the indigenous people, even though it conflicted with the treatment recommended by scientific evidence. $^{29}$ This case shows that although the biomedical worldview is privileged in Ontario legislation and official policy, the province's judicial systemcan acknowledge and demonstrate respect for nonscientific views of health and medicine.

\section{CONCLUSION}

The major distinction between CAM and biomedicine is the use of scientific evidence to revise theories of disease and treatment. Although a treatment's mechanism of action may differ according to CAM or pathophysiology, if a CAM is found to be effective in an RCT, it can be considered EBM. If a CAM lacks scientific evidence of effectiveness and safety, patients should be given this information before deciding to incorporate this therapy into their care plan, as the treatment may carry risks or confer only placebo effects. Granted, CAMs not yet disproven by the scientific method may one day be accepted by biomedicine. Cases showing promising evidence of CAM's therapeutic effects caution that rejecting CAM, without evidence of harm or lack of benefit, is narrow minded. Additionally, the subjective evidence on which CAM operates may be more useful than scientific data for determining what an event such as a sickness will mean for a patient's life.

This article explored philosophical, ethical and legal considerations surrounding physicians' recommendation of CAM to patients. The authors acknowledge that their views and beliefs have developed within the context of a predominantly biomedical education, which may offer different perspectives than those of individuals with greater exposure to traditional systems of belief. 


\section{REFERENCES}

1. Health Canada [Internet]. Ottawa: Government of Ganada Publications. Access to therapeutic products: the regulatory process in Canada. 2006 [cited 2015 Nov 10]. Available from: http://publications.gc.ca/ collections/collection_2007/hc-sc/H164-9-2006E.pdf.

2. Guyatt G, Cook D, Haynes B. Evidence based medicine has come a long way: the second decade will be as exciting as the first. BMJ . 2004;329(7473):990-1.

3. The College of Physicians and Surgeons of Ontario. Complementary/ alternative medicine. Dialogue [Internet]. 2011 Mar [cited 2015 Nov 13];(4):[8 p.]. Available from: http://www.cpso.on.ca/policies-publications/policy/complementary-alternative-medicine.

4. Public Health Agency of Canada [Internet]. Ottawa: Government of Canada Publications. Complementary and alternative health. $2008 \mathrm{Apr}$ 01 [cited 2015 Nov 14]. Available from: http://www.phac-aspc.gc.ca/ chn-rcs/cah-acps-eng.php.

5. Smith K. Articles against homeopathy - a utilitarian perspective. Bioethics. 2012;26(8):398-409.

6. Health Canada [Internet]. Ottawa: Government of Canada Publications. Pathway for licensing natural health products making modern health claims. 2012 Dec 27 [cited 2015 Nov 10]. Available from: http:// www.hc-sc.gc.ca/dhp-mps/prodnatur/legislation/docs/modern-eng. php.

7. Hammerschlag R, Zwickey H. Evidence-based complementary and alternative medicine: back to basics. J Altern Complement Med. 2011;12(4):2011-2.

8. Jagtenberg T, Evans S, Grant A, Howden I, Lewis M, Singer J. Evidence-based medicine and naturopathy. J Altern Complement Med. 2006;12(3):323-8.

9. Smith K. Homeopathy is unscientific and unethical. Bioethics [Internet]. 2012;26(9):508-12. Available from: http://doi.wiley.com/10.1111/ j.1467-8519.2011.01956.x.

10. Fantl J, McGrath M. Evidence, pragmatics, and justification. Philos Rev. 2002;111(1):67-94

11. Hughes BM. How should clinical psychologists approach complementary and alternative medicine? Empirical, epistemological, and ethical considerations. Clin Psychol Rev [Internet]. 2008;28(4):657-75.

12. Tonelli MR, Callahan TC. Why alternative medicine cannot be evidence-based. Acad Med. 2001;76(12):1213-20.

13. Guyatt G, Haynes B, Jaeschke R, Cook D, Greenhalgh T, Meade M, et al. Introduction: the philosophy of evidence-based medicine. 2005;45(8):77-89.

14. Barry CA. The role of evidence in alternative medicine: contrasting biomedical and anthropological approaches. Soc Sci Med [Internet] 2006;62(11):2646-57.

15. Ana Ning, King's College at Western University. Personal Communication.

16. Waldram JB. The efficacy of traditional medicine: current theoretical and methodological issues. Med Anthropol Q. 2000;14(4):603-25.

17. Keshet Y. The untenable boundaries of biomedical knowledge: epistemologies and rhetoric strategies in the debate over evaluating complementary and alternative medicine. Health (London) [Internet]. 2009;13(2):131-55.

18. Reiss J, Sprenger J. Scientific Objectivity. The Stanford Encyclopedia of Philosophy (Fall 2014 Edition), Edward N. Zalta (ed.) [Internet]. [updated 2014; cited 2015 Nov 17] Available from: http://plato.stanford. edu/archives/fall2014/entries/scientific-objectivity/.

19. Lui Z, Wang Y, Wu Y, Yang J. Condition and effectiveness evaluation of acupuncture for smoking cessation. Zhongguo Zhen Jiu. 2015 Aug; 35(8):851-7.

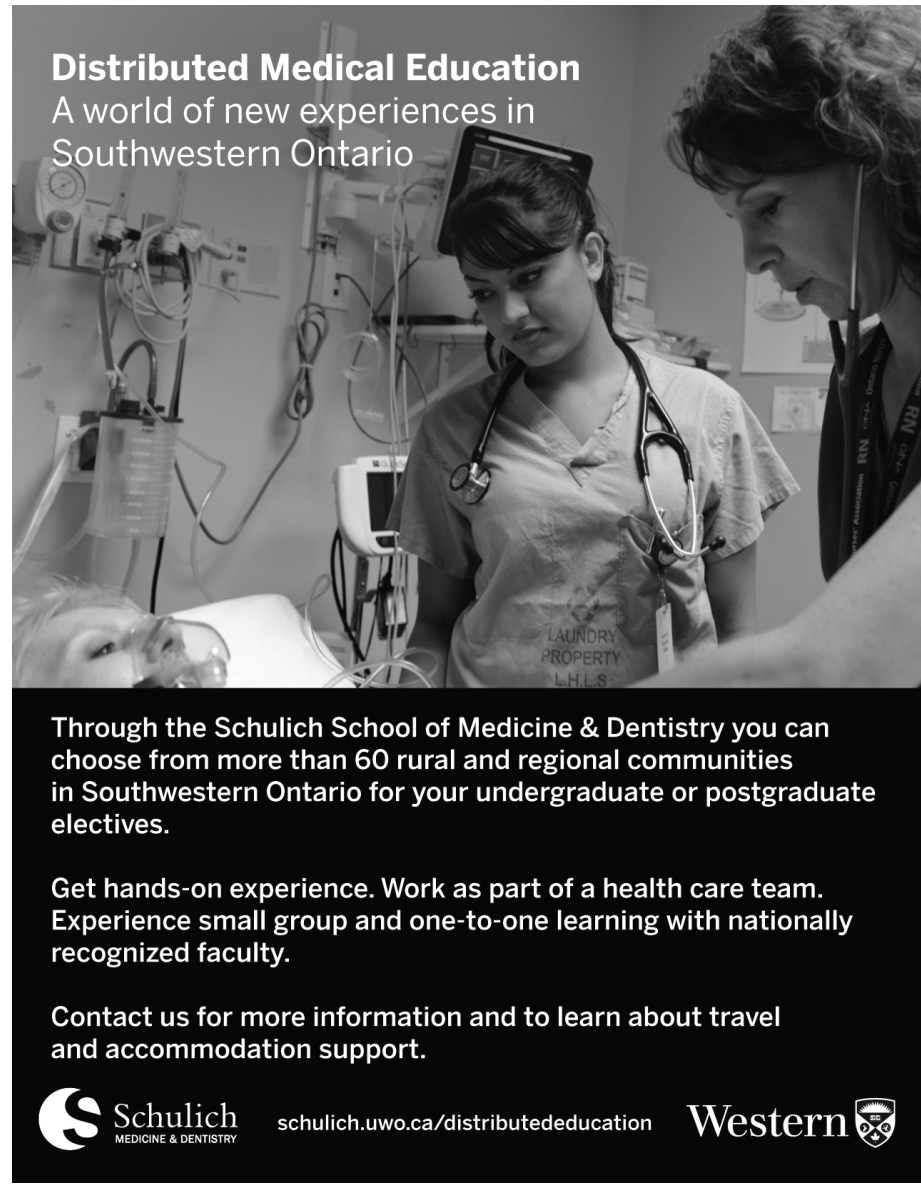

20. Beauchamp TL, Childress, JF. Principles of biomedical ethics. New York: Oxford University Press; 2009. 432 p.

21. Shaw DM. Homeopathy is where the harm is: five unethical effects of funding unscientific "remedies." J Med Ethics [Internet]. 2010;36(3):130-1. Available from: http://jme.bmj.com/cgi/doi/10.1136/ ime.2009.034959.

22. Mehta D. Ontario regulates homeopathy, greeted with skepticism by critics. The Canadian Press [Internet]. 2015 Apr 1 [cited 2015 Nov 10]. Available from: http://globalnews.ca/news/1915649/ontario-regulates-homeopathy-greeted-with-skepticism-by-critics/.

23. Urback R. Take your kid to a real doctor. National Post [Internet]. 2013 Nov 26 [cited 2015 Nov 10]. Available from: http://news.nationalpost. com/full-comment/robyn-urback-take-your-kid-to-a-real-doctor.

24. Milgrom L, Chatfield K. Is homeopathy really "morally and ethically unacceptable"? A critique of pure scientism. Bioethics. 2012;26(9):5013.

25. Briggs R. Normative theories of rational choice: expected utility. The Stanford Encyclopedia of Philosophy [Internet]. 2014 Aug 8 [cited 2015 Nov 10]. Available from: http://plato.stanford.edu/entries/rationality-normative-utility/.

26. College of Physicians and Surgeons of Ontario, Policy Statement \#3-11

27. Excellent Care for All Act, 2010, S.O. 2010, c. 14.

28. Child and Family Services Act, R.S.O. 1990, c. C.11.

29. Hamilton Health Science Corp. v. D.H. [2015] O.J. 2214. 